\title{
MONITORING AND REDUCING VEHICULAR IMPACT ON AIR QUALITY AT INTERSECTIONS: A CASE STUDY IN CHINA
}

\author{
YUN-PANG FLÖTTERÖD ${ }^{1}$, JAN TRUMPOLD ${ }^{1}$, ALEXANDER SOHR $^{1}$, HAGEN SAUL $^{1}$, \\ FENGCHENG $\mathrm{WU}^{2} \&$ YONG $\mathrm{SHI}^{3}$ \\ ${ }^{1}$ Institute of Transportation Systems, German Aerospace Center, Germany \\ ${ }^{2}$ Anhui Institute of Optics and Fine Mechanics, Chinese Academy of Sciences, China \\ ${ }^{3}$ Anhui Keli Information Industry Co., Ltd, China
}

\begin{abstract}
Air pollution has been a serious problem in China for many years. One of the main reasons for this is the rapid growth in motorized traffic which results in lots of traffic congestion in metropolitan areas. This study aims to monitor and reduce traffic's impact on air quality at intersections, with the use of the object detecting and tracking technique and traffic signal control optimization. A field campaign is executed at a selected intersection in Hefei, China and the collected emission data is used for examining and ensuring how reasonable the simulated emission productions are. The simulative approach shows that the proposed signal control method greatly increases the efficiency of the traffic operation and, at the same time, reduces the amount of emission production. A field test of the proposed method is under preparation for verifying the simulation results and will be carried out within two months.
\end{abstract}

Keywords: emission reduction, signal control optimization, object detection and tracking, VITAL-method, SUMO, optimum.

\section{INTRODUCTION}

In the recent past, air pollution has become more and more serious in China. Severe smog occurred frequently, i.e. the occurrence frequency of smog greater than $50 \%$ in Beijing and Shanghai and greater than 30\% in Guangzhou and Shenzhen. The contaminated land area even extended to 1.3 million square kilometres, containing more than 100 cities in autumn and winter occasionally. The long-lasting and large-scale extreme smog events have attracted attentions from all sectors of the society. Chinese central government has put the solution to the smog problem as the major assessment indicator of the local governments. And also, more attention has been paid to researches on traffic-induced environmental problems. It is since the rapid growth in motorized traffic often results in high congestion in the metropolitan areas in China. Some studies indicate that traffic is an important source for $\mathrm{PM}_{2.5}$ and contributed nearly to $25 \%$ of the $\mathrm{PM}_{2.5}$ production. In addition to industrial processes, vehicle exhaust has therefore become one of important emission source, especially in the mega cities, like Beijing, Shanghai in China.

Generally speaking, signalized intersections are bottlenecks in a traffic system, since the respective road capacities are limited by the given signal timing plans. The smoother the traffic can run at intersections, the less the respective congestion and the emission production will be. Vehicles are idling and produce more emissions when waiting for green times at intersections. Unnecessary waiting at intersections also results in large time loss and low traffic system performance. This study aims at monitoring and reducing traffic impact on air quality at intersections with use of the object detection and tracking technique and the traffic signal optimization. In the following, the applied methods are explained in Section 2. After that, the field study area and its current situation are illustrated in Section 3. Section 4 shows the respective analyses and results. At the end, the conclusion and the perspective are given in Section 5. 


\section{METHODOLOGY}

The applied methods to monitor and optimize traffic and environmental quality as well as the corresponding evaluation method are described below.

\subsection{Object detection, classification and tracking}

The application of video camera is the direct way to monitor the traffic quality at intersections. The collected video data can be processed for examining the corresponding traffic quality, such as traffic operation and safety, and for deriving traffic demand. In the adopted video data process of this study, the object detection software detects firstly moving objects in the traffic scene with a mixture-of-Gaussians background estimator. All incoming images are then accumulated in a multi-modal Gaussian distribution for every pixel. If a certain pixel value is not typical in this distribution, it is considered as foreground. After that, the connected foreground pixels are merged to foreground objects with a contour and a centroid point. The traffic objects of consecutive frames get associated and tracked. Furthermore, the associated object points are filtered by an extended Kalman filter which uses a non-linear motion model considering position, velocity, acceleration, rotation and rotation speed of the objects. Additionally, a smoothed trajectory of each object the tracking provides the prediction of object positions and states, which can be used to predict conflicts or collisions between traffic objects. The prediction time horizon is limited to two seconds, since farther predictions will become too vague to obtain reliable results.

Moreover, the traffic volumes and the turning movements at intersections can be derived when tracking the respective object trajectories. Such data can be used for quantitatively analysing the traffic operation, identifying critical flow groups and for other traffic analyses. The most robust way to assign a trajectory to a main traffic flow MTF (in contrast to cluster the trajectories by clustering algorithms like DBSCANS) is to use "virtual" induction loops which have to be either hit or intersected by trajectories and is used in this study.

\subsection{The VITAL-method}

Traffic signal control strongly influences the traffic and environmental quality within urban street networks. For this purpose, three signal control approaches have been applied in this paper, trying to reduce congestion, minimize waiting times and decrease vehicular emissions. These control strategies range from well-established fixed time controls for single intersections [1] to network-wide optimization schemes [2], [3], and even to decentralized self-control approaches [4]. A state of the art actuated control method based on time-gaps is also considered as a reference control method in this paper. Due to the innovation in ICT (Information and Communication Technologies) new data-sources like video capturing, wireless in-road detectors and vehicle-to-infrastructure communication (V2X) are introduced to traffic management, also offering promising options for traffic signal control. Based on this progress, a delay-based approach has been developed that utilize data from these ICT for optimized traffic signal control. In the VITAL project (Vehicle-Actuated Intelligent Traffic Signal Control) [5], [6], the validation of this new control approach (the VITAL-method) was successfully carried out in the fields of two German cities in 2016.

The concept of the VITAL-method is to use vehicles' delay times for adjusting the green times (see Fig. 1). A vehicle is considered to be delayed if its current speed on an approach is below the given maximum achievable speed, e.g. the speed limit. The delay time represents the additional travel time compared to the uninterrupted passing of an intersection, which 


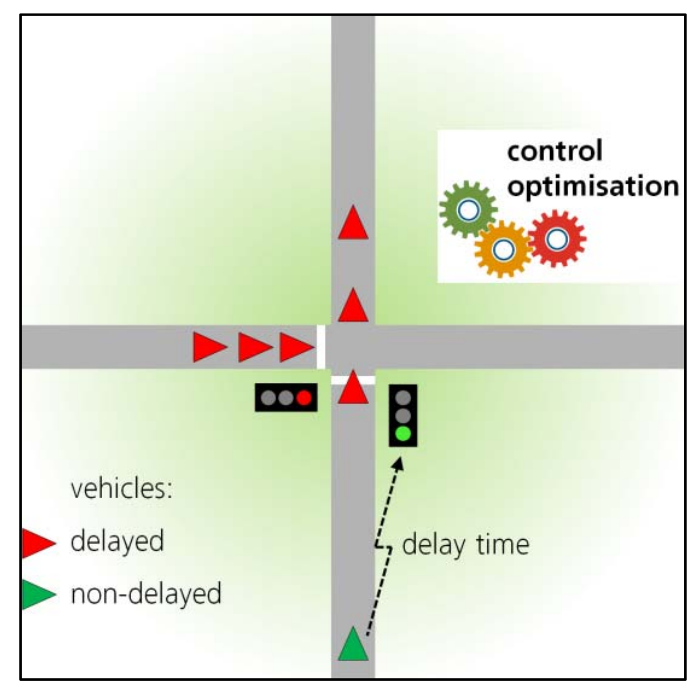

Figure 1: The concept of the delay-based VITAL-method.

includes initial deceleration delay, queue move-up time, stopped delay, and final acceleration delay [7]. By applying ICT, the delay time information can be assessed for all vehicles at an intersection and will be used for an optimized signalling. The basic idea behind this so-called delay-based control is to stop a running green phase as soon as all delayed vehicles on an approach have been served, bounded by fixed minimum and maximum green times. More details on that delay-based method and simulation results can be found in [8]-[11].

\subsection{Traffic and emission simulation}

To evaluate and verify the performance of the VITAL-method the microscopic traffic simulation SUMO (Simulation of Urban Mobility) [12] is used before the real field test in Hefei, China is undertaken. SUMO is an open-source microscopic traffic simulation and has been extensively successfully applied in different projects related to traffic emission, V2X and other diverse traffic issues. Some simulation work with SUMO for the City Hefei, China has been conducted as well [13].

The emission model HABEFA 3.1 (The Handbook of Emission Factors for Road Transport) [14], which has been already implemented in SUMO, is applied in this study. In order to reasonably represent the emission productions in China the probability distributions of vehicle types in each vehicle class, i.e. passenger car, bus and truck, have been adjusted according to the 2015 Chinese statistic report of the number of motorized vehicles. Furthermore, the minimal gap acceptance time for drivers is reduced to 1.65 seconds in the simulation in order to reflect some flexible driving manoeuvres, such as frequent lane changing, turning lanes used as through lanes or the other way around and headway shortening.

\section{FIELD STUDY}

With the consideration of the current traffic load and the available technical deployment and support City Hefei is selected for the field study. Hefei, the capital city of the province Anhui, China, has experienced the rapid economic growth and the explosive growth in urbanization 
and motorized traffic during the last few years. Such rapid changes result in serious air pollution and traffic congestion, especially during the peak periods. The annual $\mathrm{PM}_{2.5}$ concentration in Hefei is up to $83 \mu \mathrm{g} / \mathrm{m}^{3}$ in 2014. It has a slight decrease in 2015 and 2016 due to lots of control strategies have been implemented. However, the number of vehicles has increased year by year, with up to 1.5 million in 2016 . The source apportionment on fine particles shows that the traffic emission contributed nearly to $10 \%$ of $\mathrm{PM}_{2.5}$ in Hefei in 2014 . As a result, the traffic-induced environmental issues also could not be ignored along with the increase of the vehicles in Hefei. The study area and the field campaign as well as the current air quality are described in detail below.

\subsection{Study area}

\subsubsection{Location}

The ITS-test-area in Hefei, China, operated and provided by the local partner Keli, presents an ideal opportunity to implement the developed concepts and to conduct procedures under real conditions in fast growing agglomerations. The ITS-test-area covers $22 \mathrm{~km}^{2}$ and 28 intersections in the High Technology Zone in the west of Hefei. It was equipped with sensors and traffic monitoring and transportation management technology to improve the traffic, environmental and safety conditions in Hefei. All sensor-data, as well as the signal control states are available in real-time in the laboratory of Keli (see Fig. 2(a)). Furthermore, the signal control hardware can be accessed from the laboratory. So, regarding all necessary security and safety rules, new signal control algorithms can be tested. The intersection "Huangshan-Tianzhi Road" was chosen for the field test shown by the red arrow in Fig. 2(b) and in Fig. 3 in detail.

\subsubsection{Field campaign}

The field campaign has taken place from $3^{\text {rd }}$ March to $28^{\text {th }}$ March 2016. A video camera with a resolution of $1920 \times 1080$ pixels and a frame rate of $20 \mathrm{fps}$ is mounted at a height of 17 meters. It observed the intersection at a wide angle $\left(>60^{\circ}\right)$ which enables to avoid the detection problems due to the sight angle, like occlusions. The camera streams the images via WLAN to the server where the data process mentioned in Section 2.1 will be further conducted.

The Environmental Monitoring System (EMS) is mounted on the fourth floor of one of the office building closed to the intersection of Huangshan and Tianzhi Road to study the impact of traffic exhaust on environment. This system includes three types of instruments, Long-Path Differential Optical Absorption Spectroscopy (LP-DOAS), Tunable Diode Laser Absorption Spectroscopy (TDLAS) and $\mathrm{PM}_{2.5}$ monitor (see Fig. 4). LP-DOAS instrument uses a xenon lamp as light source and a UV/VIS spectrometer. The light from the high-pressure xenon lamp is collimated by the combined sending-receiving Cassegrain telescope and sent through the atmosphere. The acquisition spectra are quantitatively analysed according to the DOAS principle, and then the concentrations of trace gases are retrieved. The light path of $566 \mathrm{~m}$ of the instrument is selected with the sampling resolution of 1-3 minutes during the field campaign with the target species of $\mathrm{SO}_{2}, \mathrm{NO}_{2}, \mathrm{O}_{3}$, Benzene and Toluene and so on. The detection limit of LP-DOAS is lower than $2 \mathrm{ppb}$. A DFB laser at the center wavelength of $2334 \mathrm{~nm}$ is used as a light source for the TDLAS. With the help of an off-axis parabolic mirror, the sending and receiving light is on the same path. Based on the TDLAS principle, the CO concentration would be retrieved with the known of optical path, gas pressure and line strength. $\mathrm{CO}$ can also be used as a tracer for traffic emission in this study. The sampling resolution of the TDLAS instrument is lower than 1 minute. 
Moreover, the $\mathrm{PM}_{2.5}$ monitor uses a beta source as the detection source. The sampling is carried out by a constant flow pump. The particles are adsorbed on the surface of the filter between the beta source and detector. The number of particles can be inferred from the variations of amount of gas detector before and after counting the filter. The hourly concentrations of particles can be calculated according to the Beer-Lambert law with the known of the sampling volume. The sampling frequency of the system is 1 hour, the detection limit is $0.1 \mathrm{ug} / \mathrm{m}^{3}$ and the maximum measurement range is $0-10000 \mathrm{ug} / \mathrm{m}^{3}$.

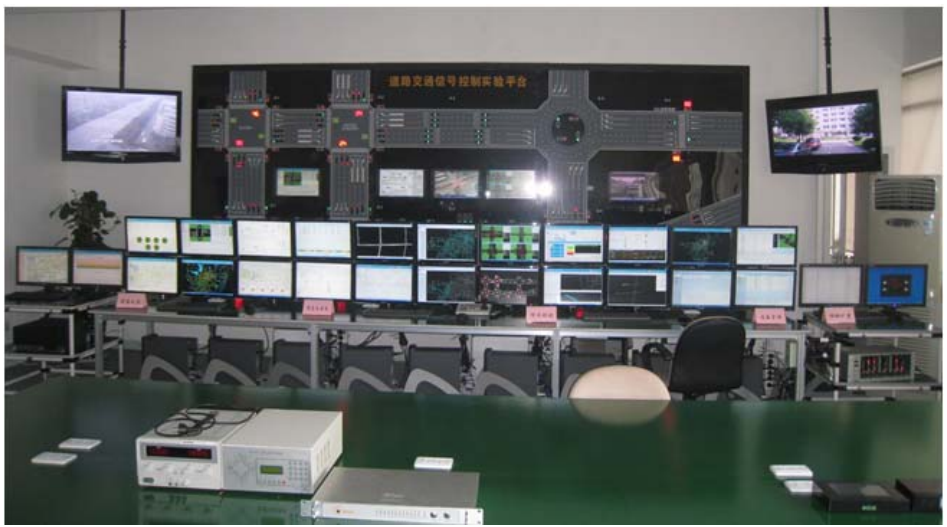

(a)

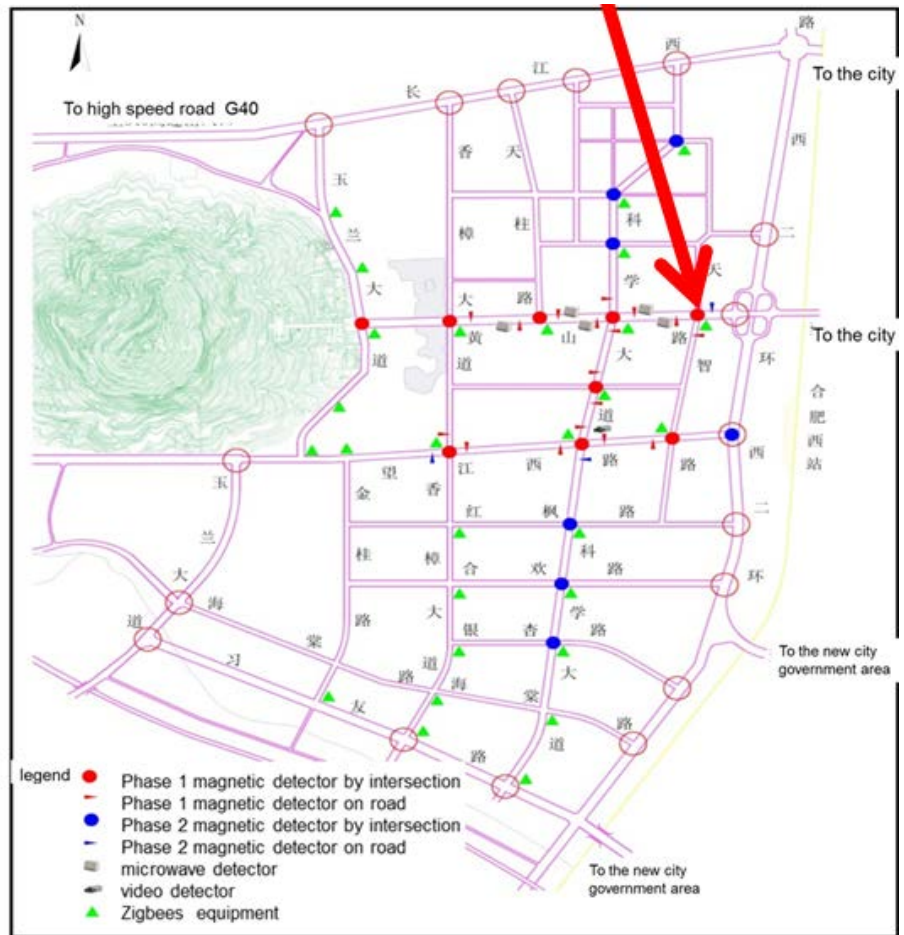

(b)

Figure 2: ITS test area Hefei and the traffic signal control experiment platform. 


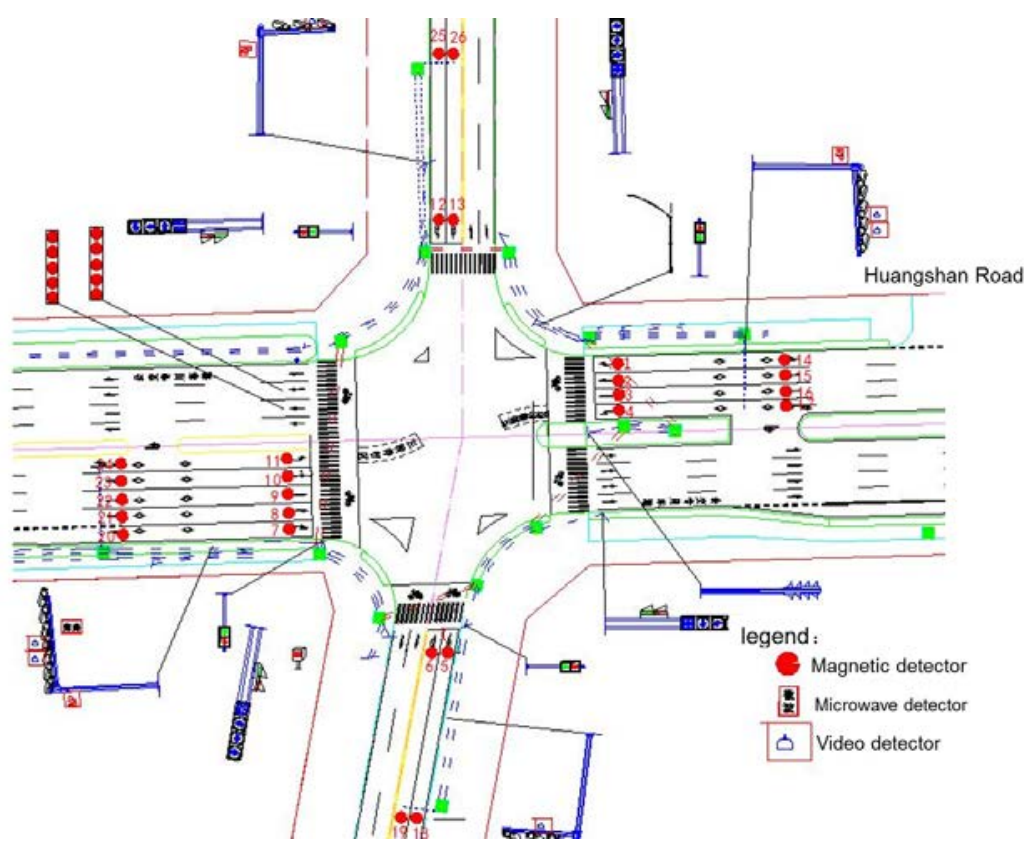

Figure 3: Location plan of the Sensors: Sensys magnet, microwave und camera in the intersection Huangshan-Tianzhi Road.
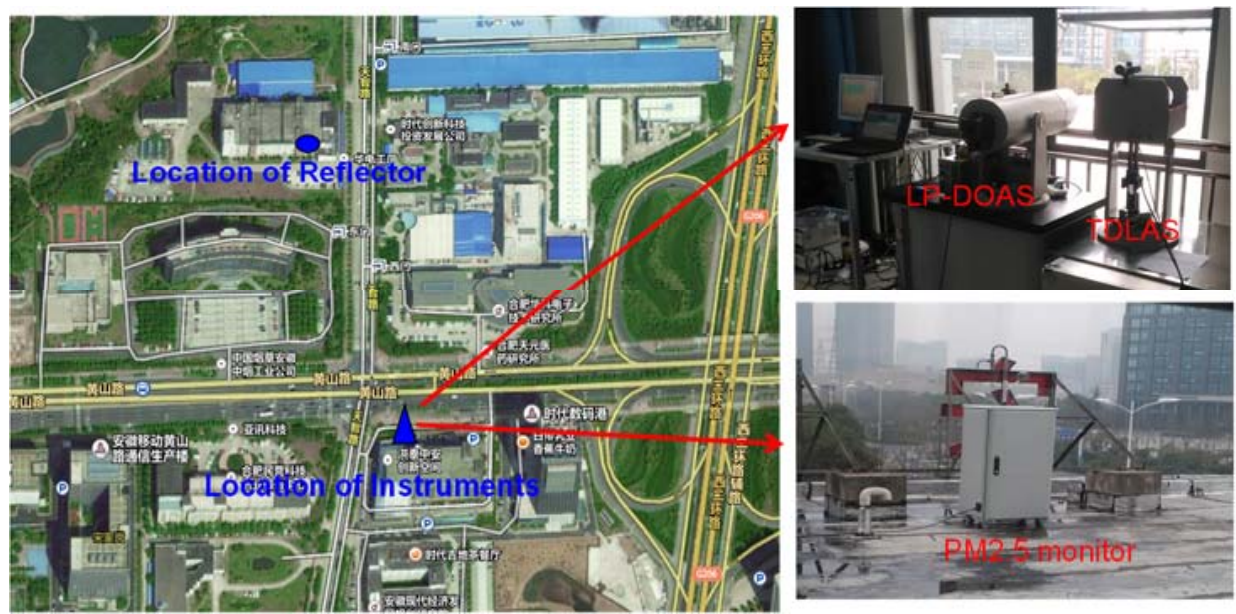

Figure 4: Set-up of the EMS.

\subsection{Current air quality situation}

During the entire measurement period, the wind directions are dominated by northeast and wind speeds are lower than $4 \mathrm{~m} / \mathrm{s}$. The results show that the typical daily variations are observed for $\mathrm{CO}, \mathrm{NO}_{2}$, Ben and Toluene, with the feature of "high at both ends and low in 
the middle" during the daytime. And two peaks in the morning and evening is mainly caused by traffic emission. However, the peak times are not identical for different air pollutants, early peak time is $07: 00-08: 00$ and late peak time is from 18:00 to 20:00 and the pollutants accumulate during the night time. The $\mathrm{NO}_{2}$ concentration is lower in weekend, but it is not obvious for Benzene and Toluene. In addition, a good correlation between Ben and Toluene shows that they come from the same source. The ratios of Ben and Toluene as well as the good correlations among Benzene, Toluene and CO suggest that traffic emission is the main source over the intersection.

The collected emission data will be used as reference data for examining if the emission data, generated from the microscopic traffic simulation SUMO, corresponds to the real measurements.

\section{SIMULATION RESULTS}

The reasonability of the simulated emissions is firstly examined with the environmental measurement data mentioned in 3.2. The performance of the proposed VITAL-method is then analysed together with the current applied signal control method (pre-timed control) and the actuated signal control method which can also adjust the corresponding green times according to the traffic demand. The traffic demand is based on the collected detector data in June and July 2016 due to the insufficient amount of traffic volume data collected during the field campaign.

\subsection{Environmental quality evaluation}

As mentioned in Section 3.1.2, the emission data was collected in a building closed to the intersection. The simulated emissions are therefore collected at the intersection with a radius of 90 meters instead of for the whole intersection network for a better comparison base. Since the amount of emissions are not only related to the amount of emission productions but also influenced by the factors such as the corresponding wind, sunlight and temperature, a direct comparison between the measurement data and the simulation data is not adequate. Due to the lack of suitable emission dispersion model the respective daily trends are compared to check if the simulation performs adequately. The intersection network and the emission collection area, i.e. the red circle, are shown in Fig. 5.

In regard to the representativeness of the results the averages of the collected data on workdays is used for analysis. The unity-based normalization eqn (1), i.e. feature scaling, is applied here to eliminate the unit effect.

$$
x^{\prime}=\frac{x-\min (x)}{\max (x)-\min (x)},
$$

where $x$ is an original value and $x^{\prime}$ is the normalized value.

According to the data collection interval the daily trends between the measured (in red) and the simulated (in yellow-green) emissions are compared at 5-minute interval for $\mathrm{CO}$ and $\mathrm{NO}_{\mathrm{X}}$ and at 60-minute interval for $\mathrm{PM}_{2.5}$. The results in Fig. 6 show that the trends of the simulated and the measured $\mathrm{CO}$ correspond to each other apparently, although some difference exists which may be due to the above mentioned weather-related factors. The amount of $\mathrm{CO}$ emission rises significantly during the peak periods when more traffic exists. The correlation between the simulated traffic and $\mathrm{CO}$ emission is therefore obvious. This result corresponds to the finding mentioned in Section 3.2. When observing the $\mathrm{NO}_{\mathrm{X}}$ results in Fig. 7, such trend consistency can be found, but only during daytime. The amount of $\mathrm{NO}_{\mathrm{x}}$ emission rises at night while the amount of the simulated NOx emission decreases 
considerably in the same time period. It may be due to that the daily air circulation has a greater influence on the amount of NOx at night. Further investigation is still needed. Fig. 8 shows that there is no apparent consistency between the daily trends of the $\mathrm{PM}_{2.5}$ emission and the simulated $\mathrm{PM}_{\mathrm{X}}$ emission. One of the reasons can be that the degree of the regional haze pollution has reached the intermediate level twice during the field campaign. One was from 2016.03.03 to 2016.03.07 and the other one is from 2016.03.13 to 2016.03.20. The data only for three workdays without the haze warning are used for analysis. Moreover, these data could also be more or less influenced by the haze pollution. Thus, further investigation is required as well.

Furthermore, the emission productions with use of three different signal control methods are analysed. Fig. 6, Fig. 7 and Fig. 8 show that both of the actuated and the proposed VITAL methods produce less $\mathrm{CO}, \mathrm{NO}_{\mathrm{X}}$ and $\mathrm{PM}_{\mathrm{X}}$ than the current pre-timed signal control method, especially during the off-peak periods. As expected, these three methods have the similar performance when the traffic demand is high during the evening peak period or very low at night. It is since there is no need to adjust the planned green times with such traffic demands.

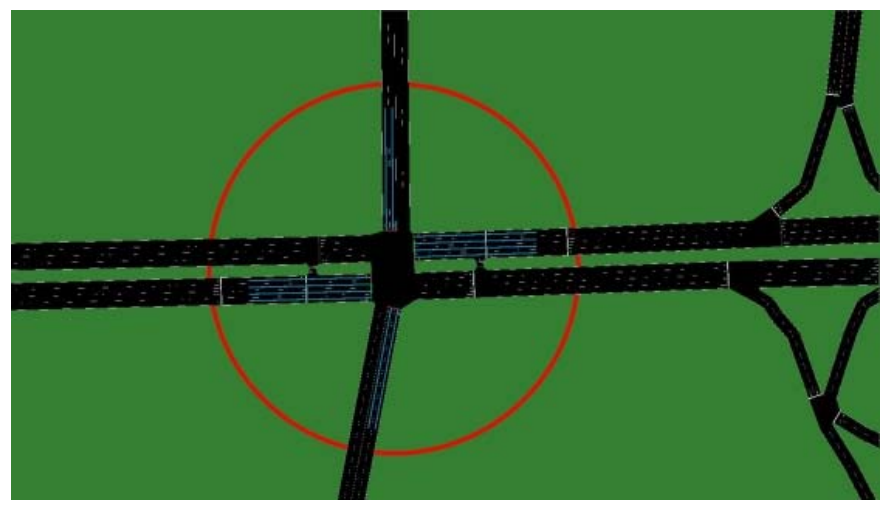

Figure 5: Layout of the simulation intersection.

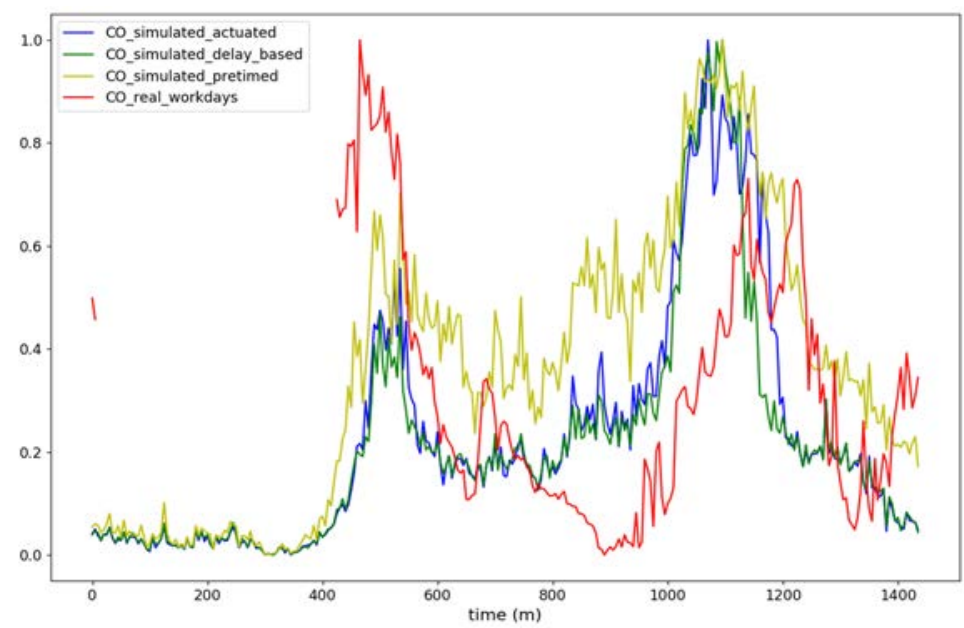

Figure 6: Normalized $\mathrm{CO}$ emissions at 5-minute interval with three control methods. 


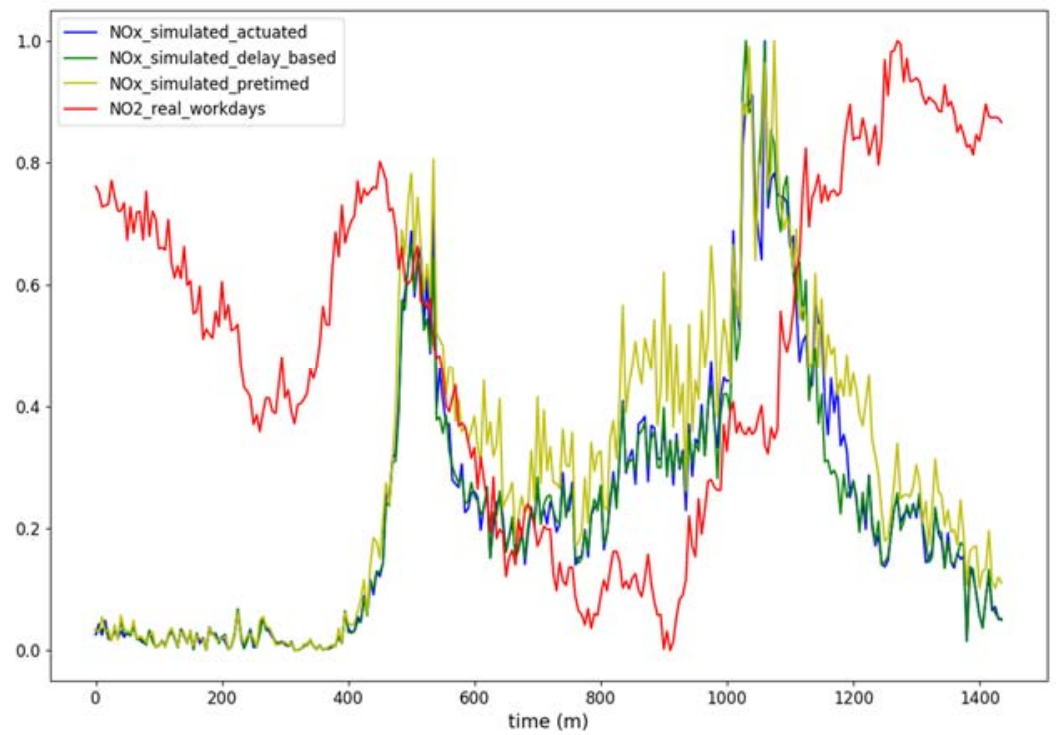

Figure 7: Normalized NOx emissions at 5-minute interval with different control methods.

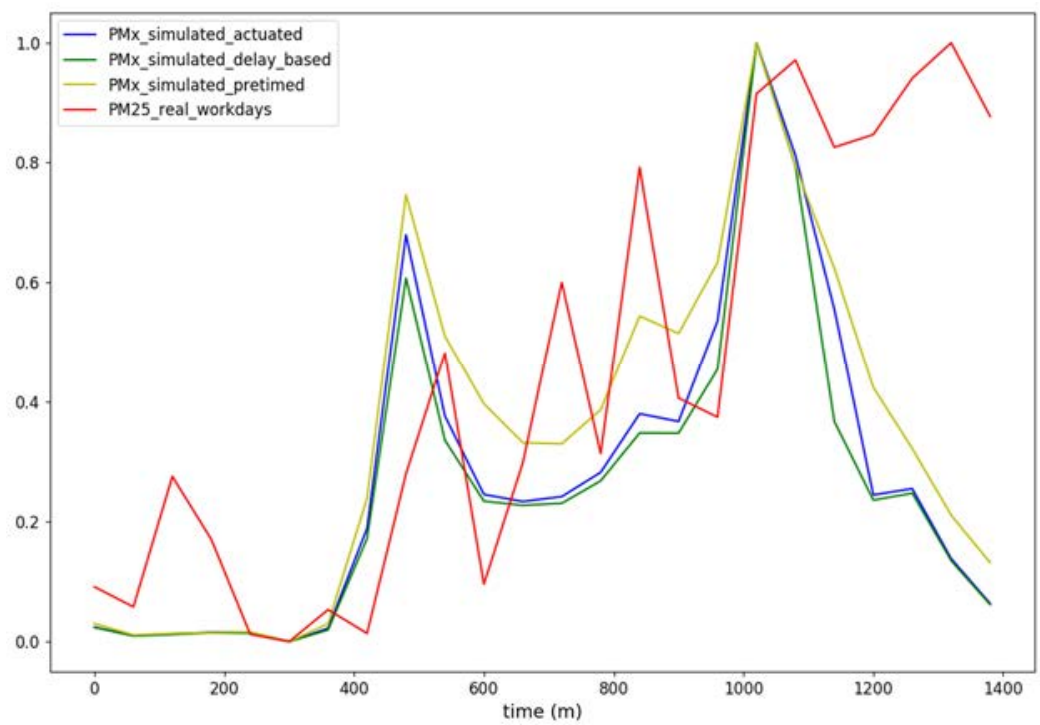

Figure 8: Normalized $\mathrm{PM}_{\mathrm{X}}$ emissions at 60-minute interval with three control methods.

\subsection{Traffic quality evaluation}

The simulation analysis has been further performed to examine the traffic quality with use of the above-mentioned three signal control methods. The first step was to model the existing control method as reference for a comparison with the VITAL and the actuated signal control 
methods. The time-gap actuated and the delay-based methods adopt the phase plans and phase sequences used in the pre-timed traffic control method. Only the green time duration will be adjusted according to the respective traffic volumes. Furthermore, the minimum and maximum green times are defined with the concern of the traffic safety and the time needed for crossing the intersection.

Table 1 shows the simulated daily traffic performance. Not only the time loss but also the waiting time and the departure delay are greatly reduced when comparing the VITAL-method with the pre-timed signal control method. The improvement is more than $45 \%$, especially the departure delay is reduced by $89 \%$. The average waiting time is reduced by $56 \%$. When comparing with the actuated method the VITAL-method delivers a better traffic quality as well, especially during the off-peak periods.

\section{CONCLUSION AND PERSPECTIVE}

The road infrastructure has been well developed and constructed in the metropolitan areas in China. However, the rapid growth in motorized vehicles results not only in serious traffic congestions but also in the environmental impacts. Regarding the transportation sustainability more focus should be put on traffic management and the related strategy development instead of building more roads. The simulative approach with SUMO indicates that the proposed signal control method is a way to manage traffic congestion and can not only increase the efficiency of traffic operation but also help to reduce the emission productions. The adopted object identification and tracking method can help to monitor the traffic operation and traffic safety at intersections. Moreover, the results show that SUMO can reproduce the daily trend of the $\mathrm{CO}$ emission properly and can be further used for the impact evaluation of different traffic management strategies.

Apparently, the traffic behaviours and the traffic regulations in China differ from those in the western countries. The VITAL-methods needs therefore to be justified regarding these circumstances. Furthermore, the existing signal control hardware and the respective interfaces may not fulfil all of the application requirements of the method. The application of an embedded industrial PC should overcome the problem of the possible hardware compatibility. As the next step, a field test for the VITAL-method will be executed within two months for the method validation. The aim of the field test is to bridge the gap between the scientific appraisal of the new approach, which are mainly based on simulation studies, and the respective commercial application on real roads.

Table 1: Simulative traffic performances at the analysed intersection on workdays.

\begin{tabular}{|l|l|c|}
\hline Signal control method & $\begin{array}{l}\text { Average performance } \\
\text { index (sec/vehicle) }\end{array}$ & Time \\
\hline \multirow{4}{*}{ Pre-timed } & Time loss & 56.29 \\
\cline { 2 - 3 } & Waiting time & 41.04 \\
\cline { 2 - 3 } & Departure delay & 73.24 \\
\hline Actuated & Time loss & 37.98 \\
\cline { 2 - 3 } & Waiting time & 24.70 \\
\cline { 2 - 3 } & Departure delay & 23.73 \\
\hline VITAL-method & Time loss & 31.18 \\
\cline { 2 - 3 } & Waiting time & 18.13 \\
\cline { 2 - 3 } & Departure delay & 8.42 \\
\hline
\end{tabular}




\section{ACKNOWLEDGEMENT}

This research was financially supported by the Helmholtz CAS joint research groups (HCJRG) of the Helmholtz association of German Research Centers. The authors are thankful to HCJRG for the support.

\section{REFERENCES}

[1] Webster, F.V., Traffic Signal Setting, Road Research Technical Paper no. 39, Department of Scientific and Industrial Road Research Laboratory: London, 1958.

[2] Hunt, P.B., Robertson, D.I., Bretherton, R.D. \& Winton, R.I., SCOOT - A Traffic Responsive Method of Coordinating Signals, TRRL Laboratory Report 1014, 1981.

[3] Lowrie, P.R., The Sydney Coordinated Adaptive Traffic System - Principles, Methodology, Algorithms. Proceedings IEE Conference on Road Traffic Signalling, pp. 67-70, 1982.

[4] Lämmer, S. \& Helbing, D., Self-Control of Traffic Lights and Vehicle Flows in Urban Road Networks. Journal of Statistical Mechanics: Theory and Experiment, P04019, 2008.

[5] Oertel, R., Erdmann, J., Mann, A. \& Wagner, P., VITAL -Vehicle-Actuated Intelligent Traffic Signal Control. Helmholtz Innovation Days 2013 - Partnering Research and Business, 2013.

[6] Oertel, R., Erdmann, J., Mann, A., Trumpold, J. \& Wagner, P., VITAL - VehicleActuated Intelligent Traffic Signal Control: Validation of two new Control Approaches in the Field. Proceedings - 11th ITS European Congress, 2016.

[7] Transportation Research Board, HCM - Highway Capacity Manual, Washington, DC: Transportation Research Board, 2010.

[8] Wagner, P., Verfahren und Vorrichtung zur dynamischen Steuerung einer Signalanlage, 2009. German Patent DE 102009033431 B4.

[9] Oertel, R. \& Wagner, P., Verfahren und Vorrichtung zur dynamischen Steuerung einer Signalanlage, 2010. German Patent DE 102010027327 B3.

[10] Oertel, R., Wagner, P. \& Lämmer, S., Verfahren zur Bestimmung einer Verlustzeit, Ver-fahren zur dynamischen Steuerung einer Signalanlage und Vorrichtung zur Bestimmung einer Verlustzeit, 2012. German Patent DE 102012220094 B3.

[11] Oertel, O. \& Wagner, P., Delay-time actuated traffic signal control for an isolated intersection. Proceedings 90th Annual Meeting Transportation Research Board (TRB), 2011.

[12] SUMO: Simulation of Urban Mobility - Wiki website, Online. http://sumo.dlr.de/wiki/. Accessed on: 10 Jul. 2017.

[13] Flötteröd, Y.P., SUMO-Cadyts calibration with limited data quality. Proceedings of the SUMO User Conference - Towards Simulation for Autonomous Mobility, pp. 155162, 2017.

[14] HABEFA, Online. http://www.hbefa.net/e/index.html. Accessed on: 10 Jul. 2017. 